\title{
Design of a Low-Noise, Fast Set-up and Low-Voltage Low-Dropout Regulator Featuring 230mA Load Current Range
}

\author{
Darshil Patel \\ Student, Department of Electronics and Communication Engineering \\ Government Engineering College, Gandhinagar \\ India \\ darshilpatel7457@gmail.com
}

\begin{abstract}
Low noise, high PSRR and fast transient low-dropout (LDO) regulators are critical for analog blocks such as ADCs, PLLs and RF SOC, etc. This paper presents design of low power, fast transient, high PSRR and high load-regulation low-dropout (LDO) regulator. The proposed LDO regulator is designed in $180 \mathrm{~nm}$. CMOS process and simulated in LTSpice and Cadence platform. The LDO proposed can support input voltage range up to $5 \mathrm{~V}$ for loading currents up to $230 \mathrm{~mA}$. Measurements showed transient time or set-up time of less than $22 \mu \mathrm{s}$, PSRR of $\sim 66 \mathrm{~dB}$ at $100 \mathrm{kHz}$ and $>40 \mathrm{~dB}$ at $1 \mathrm{MHz}$ and $0.8535 \mathrm{mV}$ of output voltage variation for a $0-230 \mathrm{~mA}$ of load variation.
\end{abstract}

Index Terms-Low-dropout (LDO) regulator, fast transient response, high power supply rejection, load regulation, low quiescent current consumption.

\section{INTRODUCTION}

As supply voltages are decreasing, advanced systems on chip (SOCs) employ increasingly lower voltage supplies, high operation speed and in part by the ever-decreasing feature size of CMOS fabrication process [1]. Further as supply voltages are decreasing and also ensuing reduced signal swing, ripple on the power supply lines start playing a dominant role in noisesensitive analog blocks such as ADCs, PLLs and RF-SOCs [2]. Thus, with extensive market growth of mobile battery-operated devices and corresponding demand for low power efficient power management circuits, low power LDO regulators with high PSRR and fast transients play a very important role and are to be focused on.

Generally, the components of an LDO regulator are error amplifier, a pass transistor and voltage sensing network mostly implemented by a resistive divider. The unity gain frequency response is mainly dominated by the parasitic poles generated at the output impedance of the error amplifier and gate capacitance of the pass transistor; and at the output due to load capacitance, load resistance and feedback resistors [2]. The dominant zero is set by the load capacitor ESR resistance and load capacitance. PSRR is dictated by the noise cancellation and high loop gain at the base of the pass transistor. And transient response time or set-up time is generally dictated by the slew-rate of the error amplifier [12]. Thus, a high gain, high slew rate error amplifier is implemented here to achieve low noise and fast set-up.

Many papers previously have focused on achieving high PSRR or fast set-up time or both of them in LDO regulator designs. [3]-[9]. The designs in [3], [4], [8] and [9] draws a large current and additional circuits increase the quiescent current consumption. The design in [5] has poor load regulation and design in [9] achieves fast set-up, draws less quiescent current but has a maximum current handling capacity of $100 \mathrm{~mA}$. Furthermore, designs in [3]-[8] are unable to achieve below 1V operation.

\section{DESIGN OF HIGH SLEW RATE OP-AMP}

Slew rate in various op-amp topologies implies that slewlimited settling can be improved only by raising the bias current and hence the power consumption [10]. Therefore, we turn our attention to "Class AB" amplifier topology as described in [10]. This topology helps us achieve high slew rate while precisely controlling the power dissipation by the virtue of temporary boost in slew rate. Fig. 1 shows the schematic of the high slew rate OP-AMP topology. 


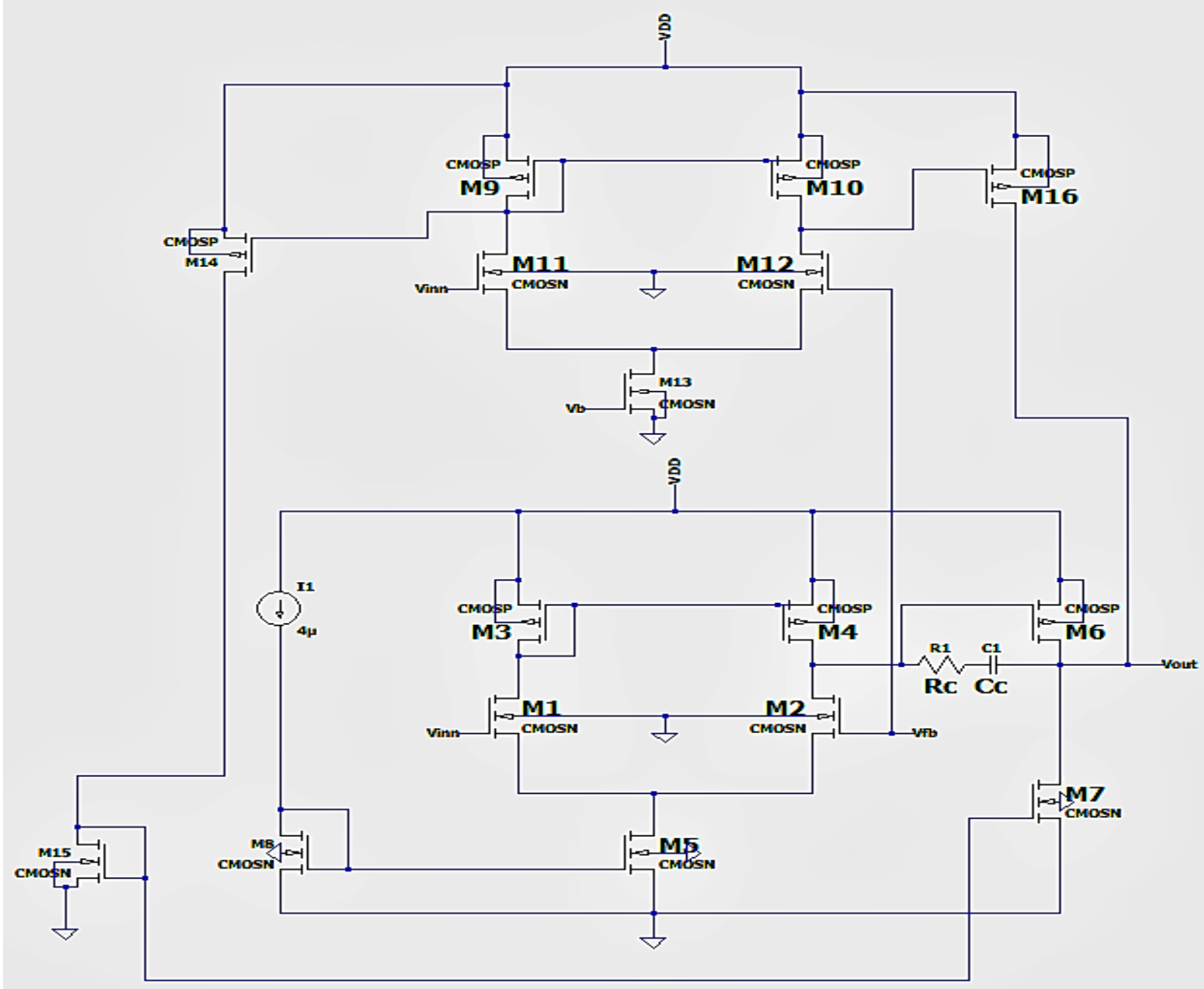

Fig. 1 Schematic of the high slew rate OP-AMP

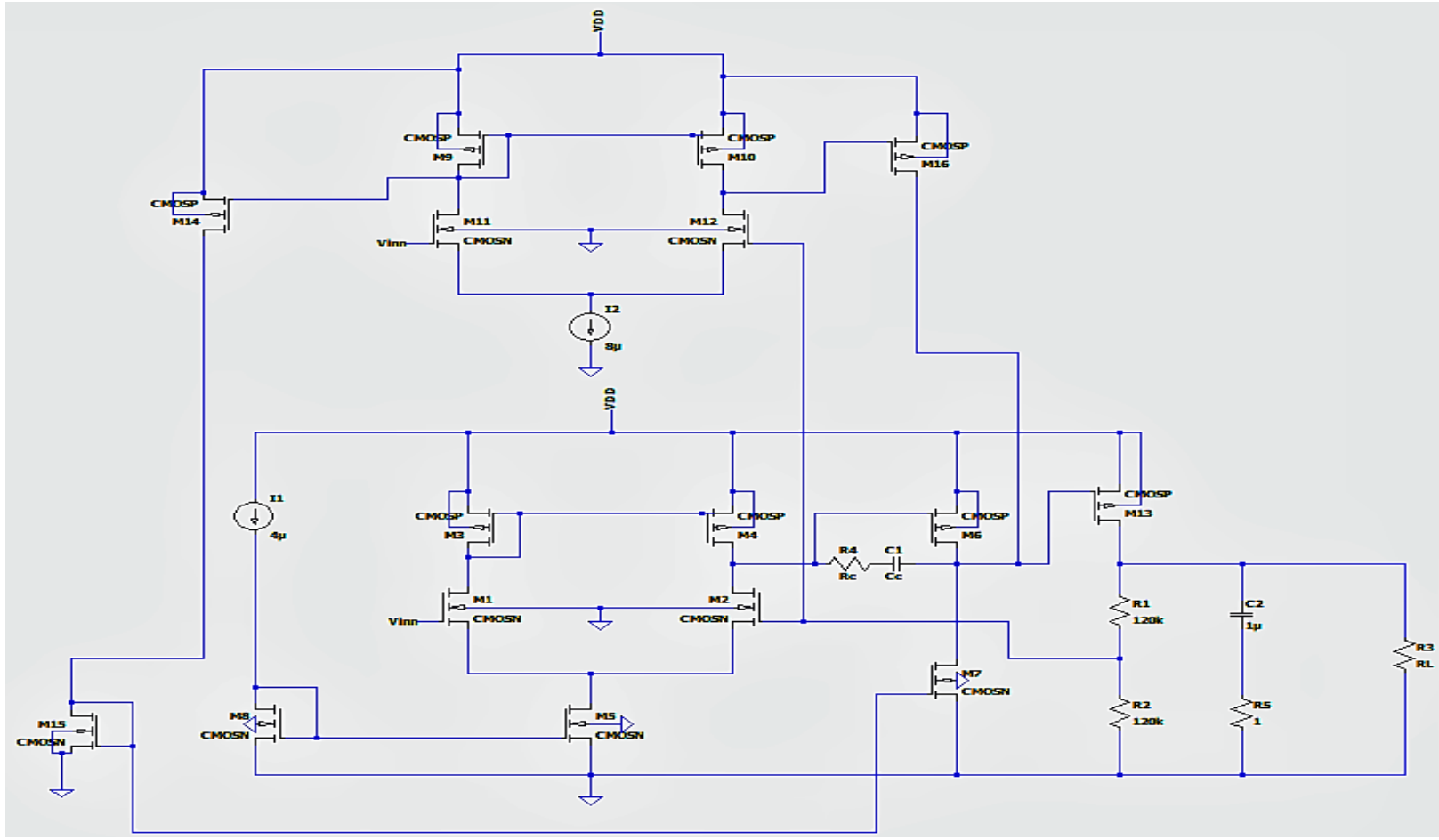

Fig. 2 Overall schematic of proposed LDO regulator 
Slewing capability is provided by transistor M16, while M6 and M7 are used for amplification. M11 and M12 senses the need of temporary boost in the slew-rate. When the input terminals Vinn and $V_{F B}$ (feedback voltage) are around same potentials during normal operation, approximately same current flows through both networks of differential pair which forces M9 and M10 to go into triode region, and as a result the drain voltages of M11 and M12 are pulled to VDD. Therefore, M16 is normally OFF during these conditions.

If $V_{F B}$ jumps up during set-up, M12 draws more current which pull it's drain terminal to negative rail and as a result M16 gets turned ON which provides large current to quickly charge the associated load capacitance [11].

As mentioned earlier, the advantage of this technique is that during large signal operation, the core amplifiers are not stressed with any large currents. In fact, in this design current consumption is decided by M5 and M17(I2) and so one can precisely control the quiescent current consumption.

\section{COMPLETE LDO REGULATOR DESIGN AND ITS ANALYSIS}

The simplified overall schematic of the proposed LDO regulator is shown in fig. 2 . It is composed of the high slew rate error amplifier earlier described, pass element, feedback network, load capacitance and load resistor. Table 1 includes all the aspect ratios of the transistors in the LDO.

The dominant pole occurs at the output node and there are three other dominant poles in the circuit which are as mentioned:

$$
\begin{gathered}
\omega_{p 1}=1 /\left[\left(r_{o 4}|| r_{o 2}\right)\left(C_{G S 6}+\left(C_{c}+C_{G D 6}\right) * g_{m 6}\left(r_{o 6} \| r_{o 7}\right)\right]\right. \\
\omega_{p 2}=1 /\left[\left(r_{o 10} \| r_{o 12}\right)\left(\left(C_{G D 16} * G_{m 16}\left(r_{o 6}|| r_{o 7}|| r_{o 16}\right)\right)\right]\right. \\
\omega_{p 3}=1 /\left\{( r _ { o 6 } \| r _ { o 7 } ) ( C _ { C } + C _ { G D 6 } ) \left(1+\frac{1}{\left.g_{m 6}\left(r_{o 6}|| r_{o 7}\right)\right)}\right.\right. \\
\left.+C_{G S 13}+A_{13} * C_{G D 13}\right\} \\
\omega_{\text {pout }}=\frac{1}{\left(R_{1}+R_{2}\right)|| r_{o 13}|| R_{\text {load }}\left[C_{G D}\left(1-\frac{1}{A_{13}}\right)+C_{L}\right]} \\
\omega_{z 1}=\frac{1}{R_{C} * C_{C}} \\
\omega_{z 2}=\frac{1}{C_{L} * R_{E S R}}
\end{gathered}
$$

Zeroes are generated by ESR of the load capacitor, load capacitor and Rc, Cc. Fig. 3(a) shows the frequency response of the LDO regulator at $0 \mathrm{~mA}$ of load current, it can be observed that the phase margin in this case is greater than 60 degrees. Fig. 3(b) shows the frequency response of the LDO regulator at $220 \mathrm{~mA}$ load current which reflects the phase margin of greater than 40 degrees.
TABLE I. TRANSISTORS' SIZES IN THE LDO

\begin{tabular}{|l|l|}
\hline Device & Parameter \\
\hline M1, M2, M11, M12 & $2.5 u / 0.5 u$ \\
\hline M3, M4 & $7 u / 0.5 u$ \\
\hline M9, M10, M14 & $0.9 u / 0.3 u$ \\
\hline M5 & $6 u / 0.5 u$ \\
\hline M6 & $87 u / 0.5 u$ \\
\hline M7 & $37.5 u / 0.5 u$ \\
\hline M8 & $3 u / 0.5 u$ \\
\hline M13 & $21 m / 0.5 u$ \\
\hline M15 & $0.6 u / 0.1 u$ \\
\hline M16 & $87 u / 0.5 u$ \\
\hline
\end{tabular}

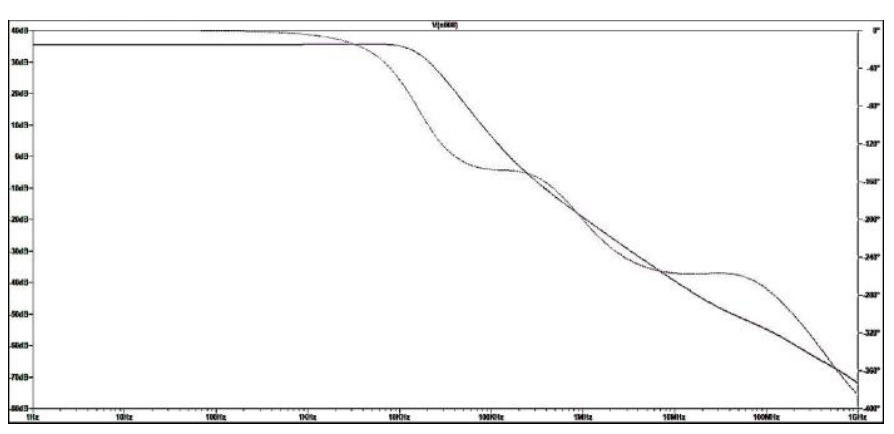

(a)

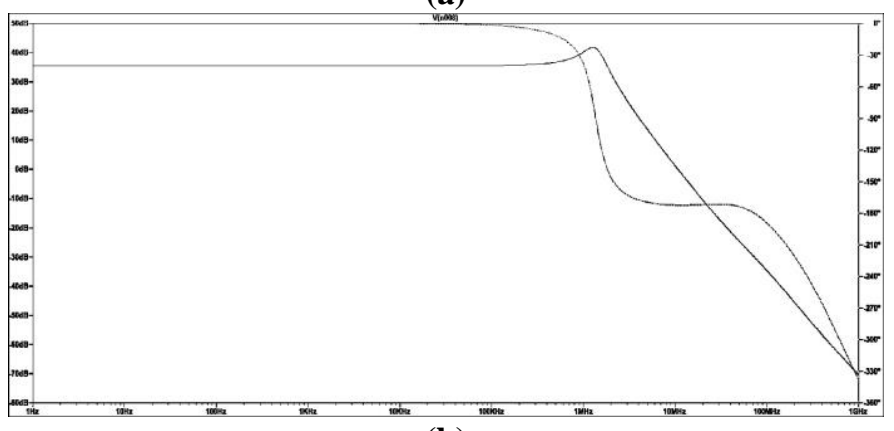

(b)

Fig. 3 Frequency response of the LDO regulator at (a) $0 \mathrm{~mA}$ load current and (b) $220 \mathrm{~mA}$ load current

The proposed LDO regulator is designed in 180nm. CMOS process technology. It is designed to deliver a maximum load current of $230 \mathrm{~mA}$. Fig. 4 shows the set-up transients. The setup time of less than $22 \mu \mathrm{s}$ can be observed. Fig. 5 shows the waveforms of the output voltage as load current changes from $0 \mathrm{~mA}$ to $230 \mathrm{~mA}$. It changes about $0.7291 \mathrm{mV}$ in this case translating to load regulation of $0.0037 \mathrm{mV} / \mathrm{mA}$. The simulated result for PSRR of the proposed LDO regulator is shown in fig. 6 and it can be seen that it achieves a PSRR of $\sim 66 \mathrm{~dB}$ at low frequencies and around $36 \mathrm{~dB}$ at high frequencies. Table 2 summarizes the performance parameters of the proposed LDO design. 


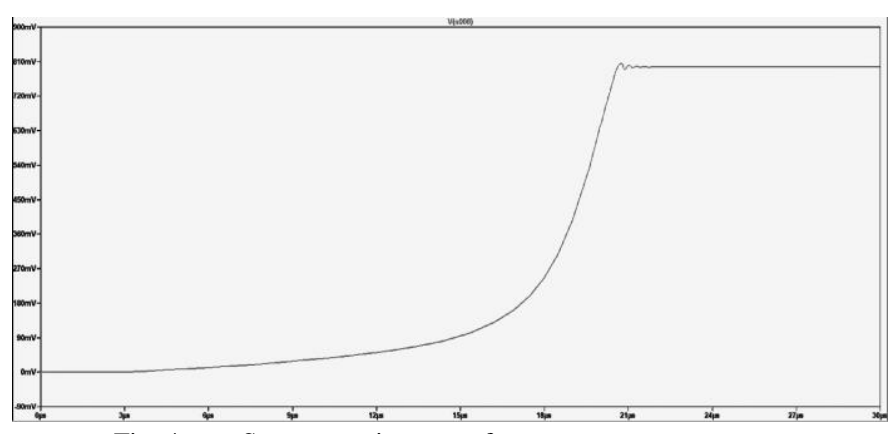

Fig. 4 Set-up transient waveform

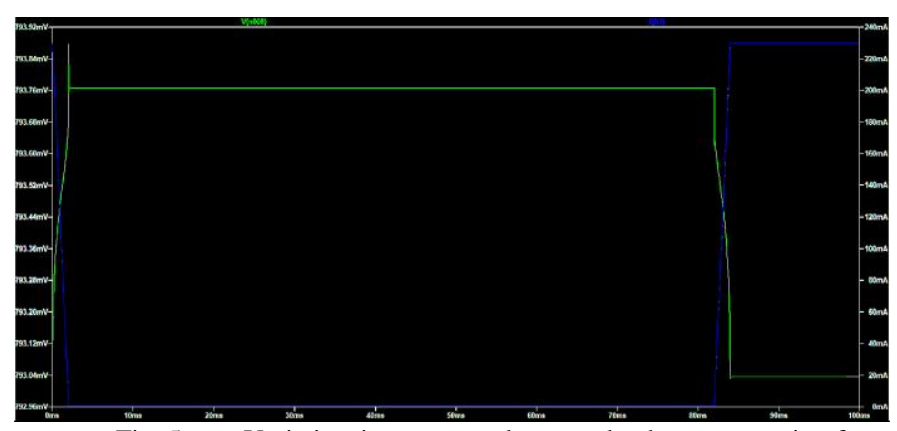

Fig. 5 Variation in output voltage as load current varies from $0 \mathrm{~mA}$ to $220 \mathrm{~mA}$

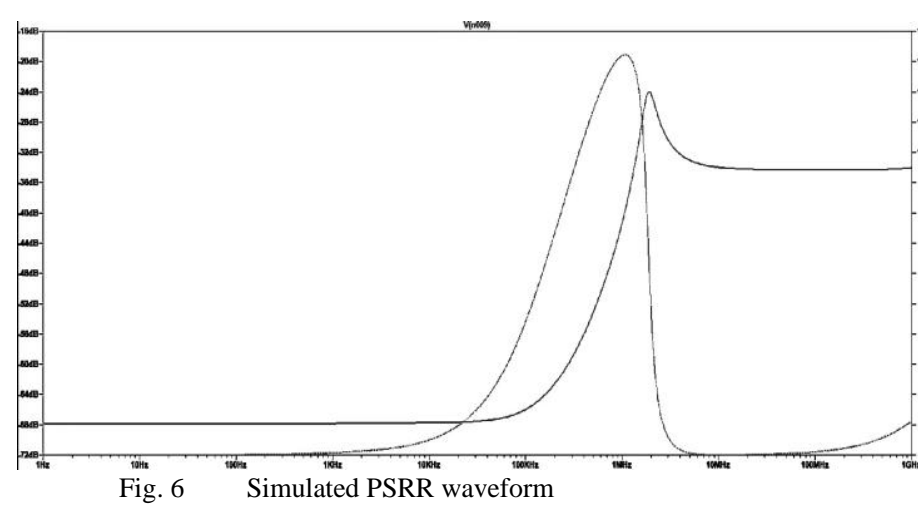

TABLE II. PERFORMANCE DETAILS OF THE PROPOSED LDO

\begin{tabular}{|l|l|}
\hline Design parameters & \\
\hline Technology(CMOS) & $180 \mathrm{~nm}$. \\
\hline Load capacitance & $1 \mathrm{uF}$ \\
\hline ESR resistance & $1 \mathrm{ohm}$. \\
\hline Quiescent current & $20 \mathrm{uA}$ \\
\hline Maximum output current & $230 \mathrm{~mA}$ \\
\hline Load regulation & $0.0037 \mathrm{mV} / \mathrm{mA}$ \\
\hline & $0.851 \mathrm{mV}(0-$ \\
Output variation & $230 \mathrm{~mA})$ \\
\hline Response time & $<22 \mathrm{us}$ \\
\hline PSRR & $\sim 66 \mathrm{~dB} @ 100 \mathrm{kHz}$ \\
\hline
\end{tabular}

\section{CONCLUSION}

This paper presents an LDO regulator design using a class $\mathrm{AB}$ amplifier topology based high slew rate operational amplifier which can achieve operation below $1 \mathrm{~V}$, has fast set- up response, low quiescent current consumption, high PSRR and good load regulation. The proposed LDO regulator was designed in $180 \mathrm{~nm}$. CMOS process to convert $1 \mathrm{~V}$ to $0.79 \mathrm{~V}$ while achieving a PSRR of $\sim 66 \mathrm{~dB}$ at low frequencies $(0-$ $100 \mathrm{kHz}$.), a good load regulation of $0.0037 \mathrm{mV} / \mathrm{mA}$ for output load variation of $0-230 \mathrm{~mA}$ and low quiescent current consumption of around $20 \mu \mathrm{A}$.

\section{REFERENCES}

[1] J. Jiang, W. Shu and J. S. Chang, "A 65-nm CMOS Low Dropout Regulator Featuring >60-dB PSRR Over 10-MHz Frequency Range and 100-mA Load Current Range," in IEEE Journal of Solid-State Circuits, vol. 53, no. 8, pp. 2331-2342, Aug. 2018, doi: 10.1109/JSSC.2018.2837044.

[2] Yongqiang Xiao, Wengao Lu, Meng Chen, Yacong Zhang and Zhongjian Chen, "A low noise, fast set-up low-dropout regulator in 65nm technology," 2012 IEEE International Conference on Electron Devices and Solid State Circuit (EDSSC), Bangkok, 2012, pp. 1-3, doi: 10.1109/EDSSC.2012.6482827.

[3] M. Al-Shyoukh, H. Lee, and R. Perez, "A transient-enhanced lowquiescent current low-dropout regulator with buffer impedance attenuation," IEEE J. Solid-State Circuits, vol. 42, no. 8, pp. 17321742, Aug. 2007.

[4] P. Hazucha, T. Karnik, B. A. Bloechel, C. Parsons, D. Finan, and S. Borkar, "Area-efficient linear regulator with ultra-fast load regulation,” IEEE J. Solid-State Circuits, vol. 40, no. 4, pp. 993940, Apr. 2005.

[5] H.-C. Lin, H.-H. Wu, and T.-Y. Chang, "An active-frequency compensation scheme for CMOS low-dropout regulators with transient-response improvement," IEEE Trans. Circuits Syst. II, Exp. Briefs, vol. 55, no. 9, pp. 853-857, Sep. 2008.

[6] A. Garimella, M. W. Rashid, and P. M. Furth, "Reverse nested miller compensation using current Buffers in a three-stage LDO," IEEE Trans. Circuits Syst. II, Exp. Briefs, vol. 57, no. 4, pp. 250254, Apr. 2010

[7] C. Chen, J. H. Wu, and Z. X. Wang, "150 mA LDO with selfadjusting frequency compensation scheme," Electron. Lett., vol. 47, no. 13, pp. 767-768, Jun. 2011.

[8] Y.-H. Lam and W.-H. Ki, "A 0.9 V $0.35 \mu \mathrm{m}$ adaptively biased CMOS LDO regulator with fast transient response," in Proc. IEEE Int. SolidState Circuits Conf., Feb. 2008, pp. 442-443, 626.

[9] C. Huang, Y. Ma and W. Liao, "Design of a Low-Voltage LowDropout Regulator," in IEEE Transactions on Very Large Scale Integration (VLSI) Systems, vol. 22, no. 6, pp. 1308-1313, June 2014, doi: 10.1109/TVLSI.2013.2265499.

[10] Behzad Razavi. 2000. Design of Analog CMOS Integrated Circuits (1st. ed.). McGraw-Hill, Inc., USA.

[11] B. Panda, S. K. Dash and S. N. Mishra, "High Slew Rate op-amp design for low power applications," 2014 International Conference on Control, Instrumentation, Communication and Computational Technologies (ICCICCT), Kanyakumari, 2014, pp. 1096-1100, doi: 10.1109/ICCICCT.2014.6993124.

[12] Ashis Maity, and Amit Patra, "A Hybrid Mode Operational Transconductance Amplifier for an Adaptively Biased Low Dropout Regulator ", IEEE Transactions on Power Electronics, vol. 32, no. 2, pp. 1245-1254, Feb. 2017. 